\title{
The analysis of cracking risk by shrinkage restraint of an alkali-activated slag mortar
}

\author{
Farah Rifai ${ }^{1,2}$, Aveline Darquennes ${ }^{3}$, Lavinia Stefan $^{4}$, Benoist Muzeau ${ }^{1}$, and Farid \\ Benboudjema ${ }^{2, *}$ \\ ${ }^{1}$ Université Paris-Saclay, CEA, DEN-Service d'Etude du Comportement des Radionucléides (SECR), \\ F-91191, Gif-sur-Yvette, France \\ ${ }^{2}$ Université Paris-Saclay, CNRS, ENS Paris-Saclay LMT, 94235 Cachan, France \\ ${ }^{3}$ INSA Rennes, 20 Avenue des Buttes de Coesmes, 35000, Rennes, France \\ ${ }^{4}$ Orano, NWM, Technical Department, 125 Rue de Paris, 92320 Chatillon, France
}

\begin{abstract}
Alkali-activated slag (AAS) binders show in general larger autogeneous shrinkage strains than ordinary Portland cement (OPC) based binders. However, AAS can be a relevant alternative to OPC, if, for example low hydration heat release and fine pores, are required. This study proposes an evaluation of the advantage of using AAS materials in small-sized or massive structures with regard to cracking risk by autogeneous shrinkage and thermal strains. A cracking risk index is calculated; this risk is defined as the ratio between stress generated by full restraint and tensile strength. All required experimental data were investigated in an OPC and AAS mortar, these are: heat release, autogeneous shrinkage, Young's modulus, tensile strength and basic creep evolutions. The material parameters of a rate-dependent model developed in 1D were then identified. Numerical simulations were then performed for different thicknesses in full-restraint conditions. These show that, as expected, basic creep is a very important material parameter to assess. Indeed, basic creep enables the significant reduction of the generated stresses. Besides, it is found that the more the structure is large (and sensitive to cracking by risk by thermal strain), the more the AAS material is becoming appropriate compared to the OPC material.
\end{abstract}

\section{Introduction}

Shrinkage and creep may significantly affect the behaviour of concrete structures. From early-age to long term, stresses occur in the restraint of autogeneous, drying and thermal strains. These strains can be restrained by concrete elements of different geometries (for example, column/slab, beam/wall). Self-restraint is due to the temperature and relative humidity gradients occurring inside concrete members. This may lead to cracking and is highly dependent upon the concrete mix (shrinkages, Young modulus, creeps etc.) and on the mechanical and ambient (temperature, relative humidity, wind etc.) boundary conditions. The consequences are multiple and depend on the density, openings, lengths, patterns etc. of the

\footnotetext{
* Corresponding author: farid.benboudjema@ens-paris-saclay.fr
} 
cracks. A reduction of global stiffness and bearing capacity may occur. Transport properties such as diffusivity and permeability may increase and therefore reduce the tightness and durability of concrete structures.

The multiple mechanisms occurring in concrete life increase complexity of the choice of the best concrete mix in the context of cracking risk by shrinkage restraint. Besides, several other constraints exist such as compressive strength and class expositions in the definition of the concrete mix. It is very time consuming to make structural finite element calculations with several concrete mixes to assess the cracking risk. In this contribution, a simple tool is proposed to compare two different mixes for the prediction of cracking risk by autogeneous shrinkage and thermal strain at early-age due to hydration heat release. A macroscopic approach is adopted. All relevant parameters are taken into account: autogenous shrinkage, Young modulus, basic creep and tensile strength evolutions. This approach uses an experimental data set (combined with identification) for the prediction of mechanical properties, shrinkage and creep evolutions. Calculations are then performed for two mortar mixes with different binders: an alkali-activated slag (AAS) and an ordinary Portland cement (OPC).

\section{Modelling approach}

The total strain is classically decomposed into several parts

$$
\boldsymbol{\varepsilon}=\varepsilon_{e}+\varepsilon_{a u}+\varepsilon_{t h}+\varepsilon_{b c}
$$

where $\boldsymbol{\varepsilon}, \boldsymbol{\varepsilon}_{e}, \boldsymbol{\varepsilon}_{a u}, \boldsymbol{\varepsilon}_{t h}$ and $\boldsymbol{\varepsilon}_{b c}$ are the total, elastic, autogeneous shrinkage, thermal strain (at early-age due to hydration heat release) and basic creep strain tensors, respectively.

In order to predict the cracking risk due to restraint free strains, stresses are calculated and compared to the tensile strength. A unidimensional problem is supposed. Stress reads then:

$$
\dot{\sigma}=E\left(\dot{\varepsilon}-\dot{\varepsilon}_{a u}-\dot{\varepsilon}_{t h}-\dot{\varepsilon}_{b c}\right)
$$

where $E$ is the Young modulus and $\sigma$ is the stress.

Crack index $I_{c r}$ can then be defined as the ratio between the stress and the tensile strength $f_{c t}$ :

$$
I_{c r}=\frac{\sigma}{f_{c t}}
$$

The more important this coefficient is, the higher the cracking risk. The autogeneous shrinkage and the thermal strain act as the motors of cracking, but it is important to take into account the Young modulus, tensile strength and creep strains, as seen in Eq. (2) and (3).

The models used to calculate all the previously defined mechanical properties and strains are briefly described below.

\subsection{Mechanical properties and autogeneous shrinkage models}

The development of the mechanical properties of concrete is related to the hydration process of cement. To express this dependency, the development of mechanical properties is defined here as a function of equivalent age. The time development of material properties is defined by the function $\beta_{c}$ that expresses gain in time with respect to the reference value of a given property: 


$$
X\left(t_{e q}\right)=X_{28}\left[\beta_{c}\left(t_{e q}\right)\right]^{n} \text { with } \beta_{c}=\exp \left[s\left(1-\sqrt{\frac{28-t_{0}}{t_{e q}-t_{0}}}\right)\right]
$$

Where $n$ is a material parameter (depending on the type of cement), $X$ is the material property $\left(X=E\right.$ for the Young modulus, $X=f_{c t}$ for the tensile strength and $\mathrm{X}=\boldsymbol{\varepsilon}_{a u}$ for autogeneous shrinkage) at equivalent age $t_{e q}$ (in days), $X_{28}$ is the material property at an equivalent age of 28 days and $s$ is a coefficient depending on the type of cement. Since temperature is assumed to be constant, the equivalent age is equal to the real age.

\subsection{Thermal strain}

The thermal strain reads:

$$
\dot{\varepsilon}_{t h}=\alpha \dot{T}
$$

where $\alpha$ is the coefficient of thermal expansion.

The temperature field is calculated by solving the heat equation, taking into account hydration heat release

$$
C \dot{T}=\nabla(k \nabla T)+L \dot{\xi} k_{b c}^{i}\left(t_{e}\right)=k_{b c_{-} 28}^{i}\left[\beta_{c}\left(t_{e q}\right)\right]^{n_{k b c}}
$$

in which $L$ is the latent hydration heat $\left[\mathrm{J}^{-3} \mathrm{~m}^{-3}\right], T$ is the temperature $[\mathrm{K}], k$ is the thermal conductivity $\left[\mathrm{W} \cdot \mathrm{m}^{-1} \cdot \mathrm{K}^{-1}\right]$ and $C$ is the volumetric heat capacity $\left[\mathrm{J} \cdot \mathrm{m}^{-3} \cdot \mathrm{K}^{-1}\right]$, which are assumed to be constant, and $\xi$ is the hydration degree calculated using:

$$
\dot{\xi}=A(\xi) \exp \left(-\frac{E_{a}}{R T}\right)
$$

where $E_{a}$ is the activation energy $\left[\mathrm{J} \cdot \mathrm{mol}^{-1}\right], R$ is the ideal gas constant $\left[8.3145 \mathrm{~J} \cdot \mathrm{K}^{-1} \cdot \mathrm{mol}^{-1}\right.$ ] and $A$ is the chemical affinity $\left[\mathrm{s}^{-1}\right]$.

\subsection{Basic creep model}

The model directly takes into account the effects of hydration. In order to reproduce the (partial) reversible part of basic creep, Kelvin-Voigt and dashpot chains are used in serial. The strains can be obtained by solving the following differential equations [1]:

$$
\tau_{b c}^{i} \ddot{\varepsilon}_{b c}^{i}+\left(\tau_{b c}^{i} \frac{\dot{k}_{b c}^{i}\left(t_{e}\right)}{k_{b c}^{i}\left(t_{e}\right)}+1\right) \dot{\varepsilon}_{b c}^{i}=\frac{\dot{\sigma}}{k_{b c}^{i}\left(t_{e}\right)} \text { and } \sigma=\eta_{b c}^{i}\left(t_{e}\right) \dot{\varepsilon}_{b c}^{j}
$$

where $\tau_{b c}^{i}$ is the characteristic time (assumed to be constant), $k_{b c}^{i}\left(t_{e}\right)$ and $\eta_{b c}^{j}\left(t_{e}\right)$ are the spring stiffness and viscosity, respectively (increasing with the equivalent age):

$$
\begin{gathered}
k_{b c}^{i}\left(t_{e}\right)=k_{b c_{-} 28}^{i}\left[\beta_{c}\left(t_{e q}\right)\right]^{n_{k b c}} \\
\eta_{b c}^{i}\left(t_{e}\right)=\eta_{b c_{-} 28}^{i} n_{k b c} \frac{t_{e q}}{t_{28}}
\end{gathered}
$$




\section{Simulation of cracking by autogeneous shrinkage and thermal strain restraint}

The mix designs of two mortars (SR0 and AAS) [2] are compared regarding cracking risk by autogeneous shrinkage. Mix designs are given in Table 1.

Table 1. Mortar mix designs ( $\mathrm{W} / \mathrm{B}=$ water to binder ratio; $\mathrm{A} / \mathrm{B}=$ aggregate to binder ratio).

\begin{tabular}{|l|l|l|l|l|}
\hline Name & Type of binder & W/B & A/B & Admixture \\
\hline SR0 & CEM I 52.5 SR0 & $0.5^{1}$ & $2^{2}$ & - \\
\hline AAS & Slag & $0.5^{1,3}$ & $2^{2,3}$ & $\begin{array}{l}\text { Sodium hydroxide }+ \\
\text { Sodium citrate }\end{array}$ \\
\hline
\end{tabular}

${ }^{1}$ Ultra Pure milli-Q (18.2 Mohm.cm) water is used

${ }^{2}$ Aggregate is a sand (0.1./1.2)

${ }^{3}$ Binder quantity is defined here as the sum of slag + admixtures

As shown in Eq. (2) and (3), the cracking risk by autogeneous shrinkage restraint is highly related to the evolution of autogeneous shrinkage strains, thermal strains, Young's modulus, basic creep and tensile strength. All these material properties have been experimentally characterised and all the material parameters have been identified [2].

In order to calculate the cracking index (Eq. (3)), one supposes a full-restraint case, i.e., $\varepsilon=0$. The objective is therefore to compare the ability of the two considered mixes to crack (Table 1). Basic creep is assumed to be similar in compression and tension, due to the lack of data and lack of scientific evidence [1,3]. A finite difference scheme is adopted. The convergence was checked by performing several numerical simulations using different time steps.

\subsection{Restraint by autogeneous shrinkage}

Only autogeneous shrinkage is taken into account. The evolution of the cracking index (related to autogenous shrinkage restraint only) with respect to equivalent time is plotted in Fig. 1. As seen in Fig. 1, it is very important to take basic creep strains into account for the assessment of cracking risk (as expected). When basic creep is taken into consideration, the cracking index is divided by a factor of approximately two.

Moreover, the numerical simulations (Fig. 1) enable the ordering of the mixes with respect to their ability to crack or not due to autogeneous shrinkage restraint ( 1 is the best mix):

- SR0

- AAS

This ordering was not straightforward without the performance of numerical simulations. For example, even if the basic creep strains of the AAS mortar are more important than the other mixes, its cracking index remains significantly more important. Its large autogenous shrinkage and slightly slower tensile strength is not counterbalanced by its lower Young modulus and higher capacity for creep. 


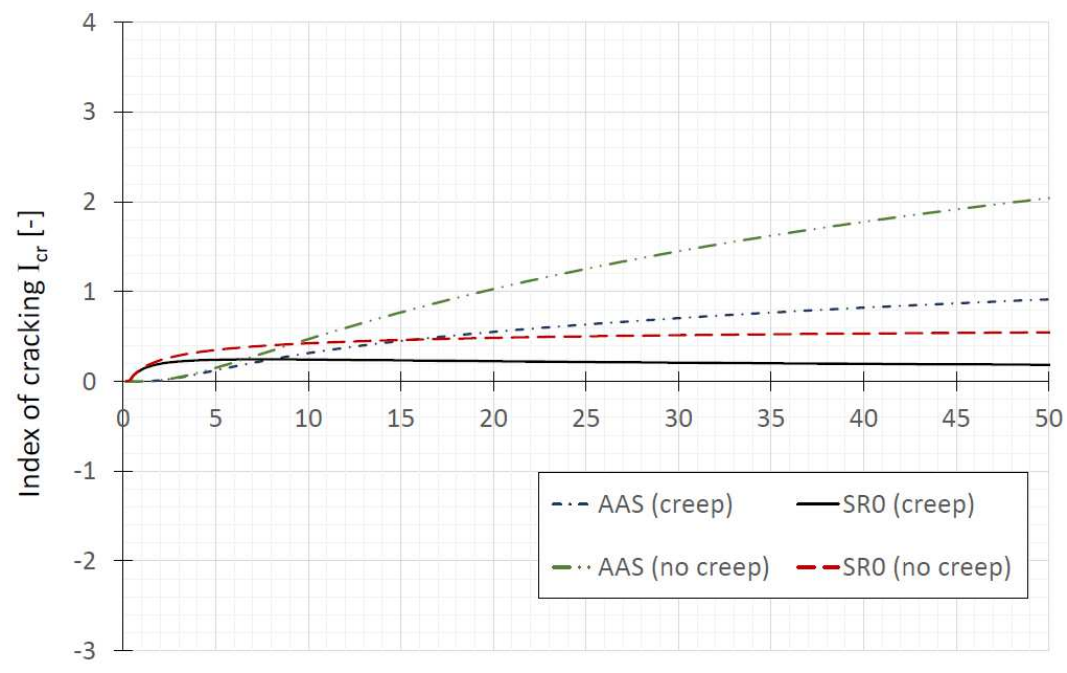

Equivalent time $t_{\text {eq }}$ [days]

Fig. 1. Evolution of cracking index with respect to equivalent time: effect of basic creep and mix design. Only autogeneous shrinkage is taken into account.

\subsection{Restraint by thermal strain at early-age and autogeneous shrinkage}

Thermal strains are also taken into account. Initial and external temperatures are equal to $20^{\circ} \mathrm{C}$. Two different thicknesses $(0.5$ and $1.5 \mathrm{~m})$ are considered. The evolution of cracking index (related to autogenous shrinkage and thermal strain restraint) with respect to the equivalent time is plotted in Fig. 2.

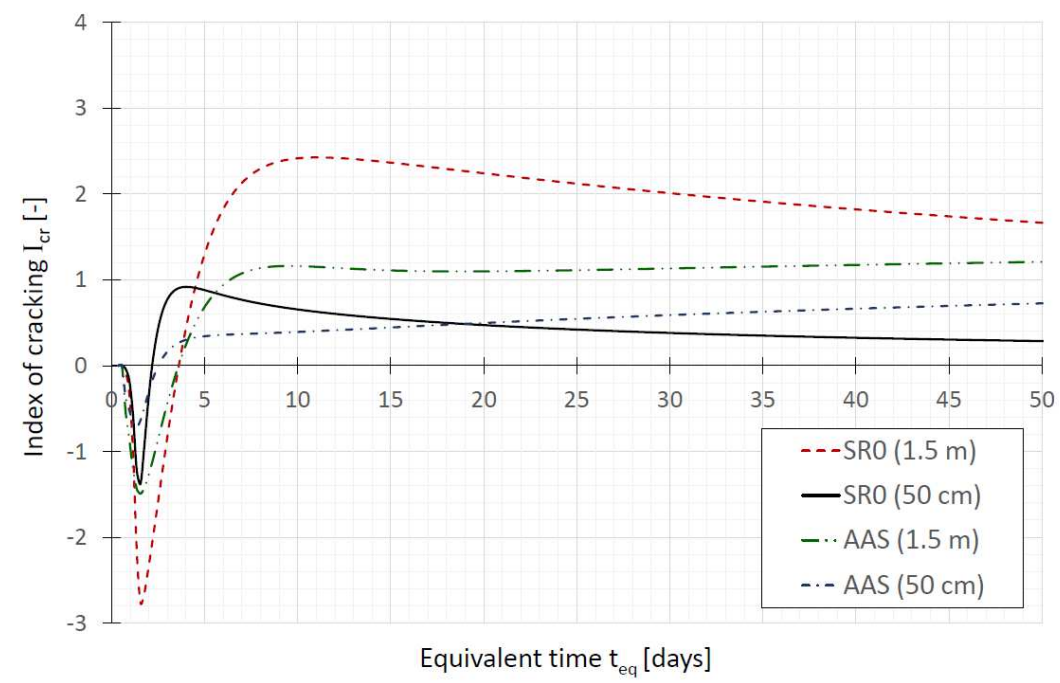

Fig. 2. Evolution of cracking index with respect to equivalent time: effect of basic creep and mix design - autogeneous shrinkage and thermal strain are taken into account. 
Again, the numerical simulations (Fig. 2) enable the ordering of the mixes with respect to their ability to crack or not due to both autogeneous shrinkage and thermal strain restraint ( 1 is the best mix):

- AAS

- SR0

This ordering was not straightforward without the performance of numerical simulations. For example, the SR0 mortar mix has the maximal heat released, i.e., maximum thermal strain, but the lowest autogeneous shrinkage.

\section{Conclusion}

The study presented here was essentially related to the comparison of cracking risk at an early-age by autogeneous shrinkage restraint between different mix designs of cement and alkali-activated slag in autogenous conditions. Numerical simulations were performed after the identification of material parameters based on the undertaken experiments. The numerical results underline several results allowing the determination of which mix is more appropriate regarding cracking risk by autogeneous shrinkage and thermal strain restraint.

The use of alkali-activated material increases risk of cracking by autogeneous shrinkage as SR0 mortar mix shows a decrease of the cracking index with respect to time. However, for both 0.5 and $1.5 \mathrm{~m}$ thicknesses, the use of alkali-activated material significantly decreases the risk of cracking by thermal strain at an early-age (due to hydration heat release). Besides, as expected, creep strains allow the reduction of the cracking risk in all the calculations, and should be taken into consideration.

In perspectives, it is important to take drying shrinkage into account when considering the service-life of concrete structures.

\section{References}

1. A. Hilaire, F. Benboudjema, A. Darquennes, Y. Berthaud, G. Nahas, Nucl. Eng. Des. 269, 222-230 (2014)

2. F. Rifai, Immobilisation de déchets magnésiens dans un matériau alcali-activé : Etude expérimentale et numérique, (PhD thesis, ENS Paris-Saclay, 2017)

3. J. Brooks, A. M. Neville, Mag. Concrete Res. 29, 131-141 (1977) 